\title{
Analysis of 5G Non-Orthogonal Technology Application on Smart Grid
}

\author{
Gang Liu ${ }^{1 *}$, Huixian $\mathrm{Lu}^{1}$, Mingsong Jin ${ }^{1}$, Peng $\mathrm{Yu}^{1}$ \\ ${ }^{1}$ Smart Shine Microelectronics Technology Co. Ltd, Beijing, China
}

\begin{abstract}
Fifth generation $(5 \mathrm{G})$ wireless communication networks are being deployed worldwide at present, which not only enhances transmission ability with wide bandwidth for wireless mobile communication, but also enables $5 \mathrm{G}$ to promote a new digital, network and intelligence for all industry. Power communication has unique requirement and feature for itself, and with more and more power and other energy terminal devices need to access network, the current air access technology is difficult to satisfy the requirements. The $5 \mathrm{G}$ had carried out many key technology researches, including air interface and network technology, which establish solid basement for $5 \mathrm{G}$ standard and $5 \mathrm{G}+$ industry application. The article focuses on $5 \mathrm{G}$ Non-Orthogonal Multiple-Access (NOMA) technology, especially PDMA, and illustrates technology application scheme with power collection requirement as an example. It can be seen that NOMA can enhance network capability greatly for power communication, and improve transmission cover ability.
\end{abstract}

\section{Introduction}

In order to create a full-service ubiquitous power Internet of Things and build smart enterprises, electric power companies will comprehensively use new information and communication technologies such as "Big-Cloud-ThingMove-Intelligence" to penetrate and deeply integrate with the new generation of power systems, and connect human, machines and things of each link in energy and power production and consumption online in real time. At the terminal layer, it shows the connection ability of the Internet of Everything; at the network layer, it shows the ubiquitous and all-time communication ability; and at the platform layer, it shows the ability to control panoramic equipment and data.

The electricity consumption information collection (abbreviation: usage and collection) system is an important part of the smart grid. The physical structure of the traditional collection system is composed of the collection master station layer, the remote communication channel layer, the collection equipment layer, the local communication channel layer and the electricity meter layer. This multi-segment communication architecture, especially the local communication network, limits the frequency of electricity information collection, resulting in poor service scalability. At the same time, the existing communication access technology cannot withstand the access of more and more business terminals to the network, and it is difficult to meet the needs of future smart energy consumption information collection and business analysis such as smart meters.

As a new-generation cellular mobile communication technology, the fifth-generation mobile communication technology $(5 \mathrm{G})$ has the characteristics of high bandwidth, low latency, and large connections, and can provide three types of typical business scenarios: eMBB enhanced mobile broadband, mMTC massive IoT, uRLLC ultrahigh reliability and low latency, which meet the business needs of power and industrial Internet of Things.

The 5G system provides 20 times the 4G network capacity, 10 times the user experience rate, 1/10 air interface delay, 3 times the spectral efficiency improvement, and a connection density of one million/ $\mathrm{km} 2$ through several key technologies and a new network architecture.

This article first introduces $5 \mathrm{G}$ system overview, application scenarios and key technologies, and then analyzes 5G key technologies, focuses on non-orthogonal multiple access capabilities, and then elaborates the value, feasibility and solutions of non-orthogonal technologies in the application of power information collection systems. Finally, the development trend of $5 \mathrm{G}+$ power and integrated energy system is summarized.

\section{Communication requirements and characteristics of smart grid}

Smart Grid enables multiple applications to operate over shared network resources, providing monitoring and control of a utility system. As an important infrastructure supporting the development of smart grids, the power communication network ensures the safety, real-time, accuracy and reliability requirements of various electric power services. On the side of the distribution communication network, due to the wide variety of points, a large number of devices need to be monitored or

"e-mail: liugang2@sgitg.sgcc.com.cn 
controlled in real time, and information is frequently exchanged in two directions. With the rapid development of large-scale distribution network automation, advanced metering, distributed energy access, and two-way user interaction, the communication needs of various grid equipment, power terminals, and power customers have exploded, and there is an urgent need to build a safe and reliable, access flexible, two-way real-time interactive "ubiquitous, full coverage" distribution communication access network, and adopt advanced, reliable, stable and efficient emerging communication technologies and systems to support.

The following figure shows the new requirements for future power communication systems in the four links of power generation, transmission, distribution and power consumption, thus forming a fully connected smart grid. New wireless technology is the key to realizing the number of connections from thousands to billions, and the coverage from trunk lines to full coverage.
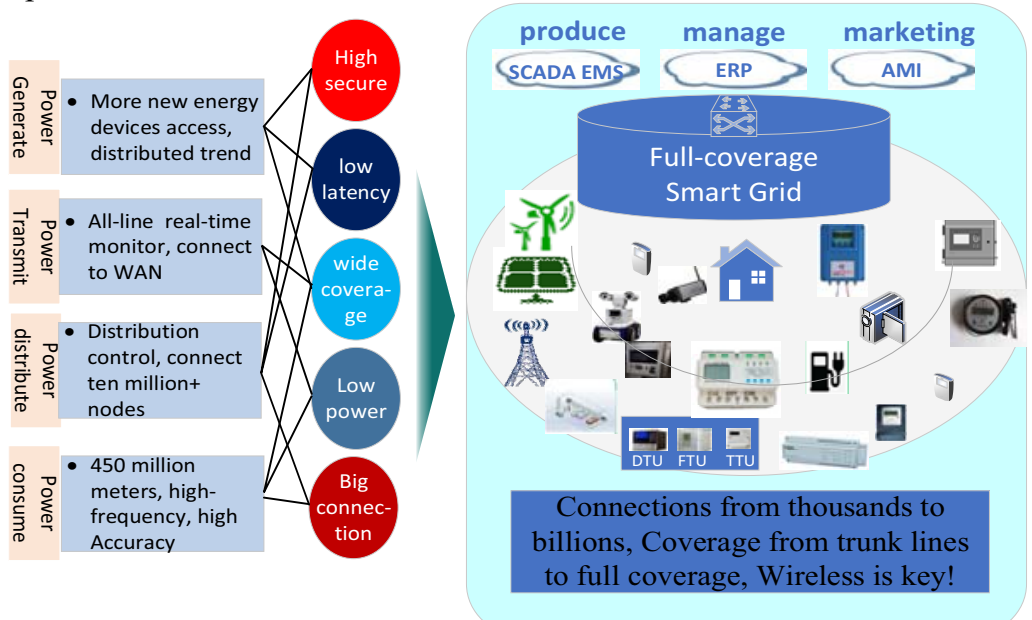

Figure 1. power communication demand characteristics

\section{$3 \mathbf{5 g}$ system and technology}

\subsection{Overview of $5 \mathrm{G}$ system}

The mobile communication system has experienced more than 30 years of development, and its application has become very popular. With the advancement of technology, its application will become more extensive. $5 \mathrm{G}$ will meet the future demand for mobile data growth exceeding 1,000 times in a sustainable way, and will provide users with multi-scene consistent services such as fiber-like access speeds, "zero" latency experience, connection capabilities of hundreds of billions of devices, ultra-high traffic, ultra-high connection density, and ultrahigh mobility.

Rel-15, the first phase of 5G, was frozen in Q3 2018. As the second phase of $5 \mathrm{G}$ standard version, Rel-16 mainly focuses on vertical industry applications and the improvement of the overall system. Its main functions include $5 \mathrm{G} \mathrm{V} 2 \mathrm{X}$ for the field of smart car transportation. The enhancement of industrial IoT and URLLC can fully replace wired Ethernet in factories. 5G NR capabilities, such as time-sensitive networking, including LAA and 5G NR in independent unlicensed bands. ASN.1 freezes the Rel-16 version in June 2020. In December 2019, the RAN\#86 meeting finally confirmed the approval of the content of Rel-17, and the formal formulation of Rel-17 has begun. It is expected that the Rel-17 specification will be frozen in June 2021.

$5 \mathrm{G}$ represents the evolution and revolution of mobile technology and can achieve many high-level goals released so far. It is generally believed that $5 \mathrm{G}$ is a generation of wireless technology that allows cellular networks to expand to new uses and vertical markets. 5G technology can also allow cellular networks to enter the world of machines, used for driverless cars, etc., and used to connect tens of billions of industrial sensors and various wearable electronic devices. $5 \mathrm{G}$ is the general term for solutions that integrate the technological evolution of existing wireless access technologies (including $2 \mathrm{G}, 3 \mathrm{G}$, $4 \mathrm{G}$, and $\mathrm{WiFi}$ ) and new complementary wireless access technologies. $5 \mathrm{G}$ will be a truly converged network. This unified standard will provide high-speed, safe and free connections between people, people and things, and things. The Internet of Things will greatly expand $5 \mathrm{G}$ services. Networks such as mobile medical care, Internet of Vehicles, smart home, and industrial control will promote the explosive growth of the Internet of Things. Massive devices will be connected to the network to realize the true "Internet of Everything".

\subsection{G application scenarios}

From the perspective of different information interaction objects, the current $5 \mathrm{G}$ applications are divided into three categories: enhanced mobile broadband (eMBB), massive machine communication (mMTC), and ultra-reliable lowlatency communication (URLLC). The eMBB scenario refers to the further improvement of user experience and other performance on the basis of the existing mobile broadband business scenario, mainly in pursuit of the ultimate communication experience between people. Both mMTC and URLLC are application scenarios of the Internet of Things, but their respective focuses are different. mMTC is mainly the information interaction 
between people and things, while URLLC mainly reflects the communication needs between things. The following

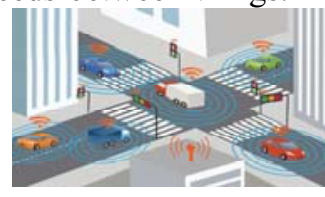

$\mathrm{V} 2 \mathrm{X}$

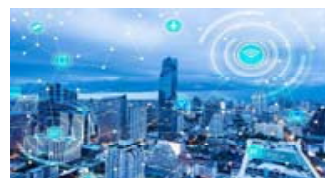

Intelligent city

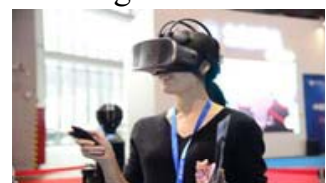

VR/AR

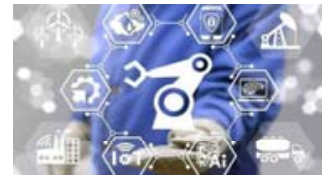

Industrial internet

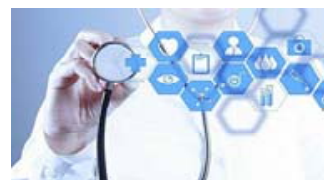

Medical care

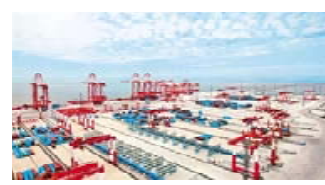

Intelligent port

Figure 2. 5G application scenarios

$5 \mathrm{G}$ has become the forerunner of the digital strategy of countries around the world, and is the infrastructure for the development of national digital and information technology. At the same time, more vertical industries such as electric power, automobiles, and industrial manufacturing have deeply participated in $5 \mathrm{G}$ standards and guided the formulation of standards in their respective fields, so that $5 \mathrm{G}$ technology can better serve all vertical industries. Focusing on the field of smart grid, especially in the link of smart power distribution and utilization, $5 \mathrm{G}^{+}$ technology provides a better solution for the "last mile" wireless access communication coverage of the distribution communication network. Intelligent distributed distribution automation, advanced metering, distributed energy access and other businesses can leverage $5 \mathrm{G}+$ to achieve greater technological breakthroughs in the future.

The possible wide application of $5 \mathrm{G}$ technology in the vertical field represented by electric power can be divided into three levels: one is to use the operator's $5 \mathrm{G}$ mobile communication access network, and to provide services by assigning different core network slices to different vertical fields; the other is that each vertical field is designed to design a dedicated network based on the $5 \mathrm{G}$ mobile communication network; the third is to adopt or learn from some advanced communication technologies researched and used by $5 \mathrm{G}$ in response to the specific new needs and characteristics of the vertical field. This article mainly analyzes the application of technology from the perspective of 5G wireless air interface technology, and taking the power collection business requirements as an example.

\subsection{G key technologies}

The 5G system supports multiple services and application scenarios, and it is difficult to use a single wireless air interface technology or wireless system to meet all needs and goals. Therefore, $5 \mathrm{G}$ initiated a number of researches on key technologies in the early stage, including air interface transmission technology and wireless network technology. This article focuses on the application of air interface transmission technology in power consumption systems.

$5 \mathrm{G}$ wireless transmission technologies mainly include the following six categories:

Wireless transmission technologies

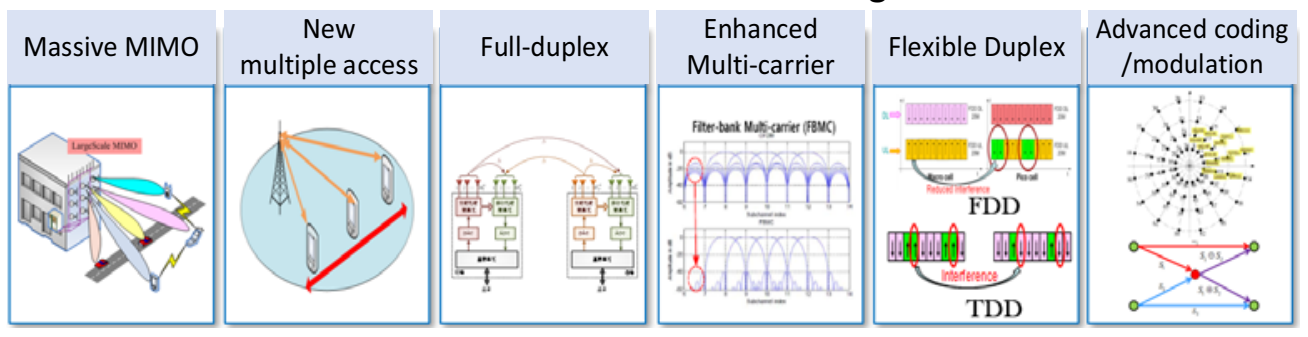

Figure 3. 5G wireless transmission technologies

\section{(1) Large-scale antenna technology}

Multiple antennas have huge advantages in improving peak rate, system frequency band utilization efficiency and transmission reliability. The performance gain of large-scale antenna technology comes from the spatial freedom of multi-antenna channels. With the rapid increase in data transmission services and the number of users, applications related to high-speed transmission and public connections will generally adopt multi-antenna technology. At present, the technology has been incorporated into the $5 \mathrm{G}$ technical specifications, and its research has been relatively in-depth, and it has also been widely used in mobile communications and industrial industries, such as AR/VR, high-definition video surveillance and hotspot area coverage.

(2) New multiple access technology

Multiple access technology has become a landmark technology for the upgrading of past mobile 
communication systems. The orthogonal multiple access method is adopted commonly in past mobile communication systems, including R15 and R16 versions for mobile broadband and ultra-reliable low-latency $5 \mathrm{G}$, but the orthogonal method limits the degree of freedom of wireless resources. For communication facing a large number of machines, the system cannot achieve optimal spectrum utilization. From the perspective of multi-user information theory, non-orthogonal multiple access can achieve significant performance gains and will greatly increase system capacity.

(3) Full-duplex technology

Duplex technology is a technology for the realization of two-way communication between communication nodes, and the same-frequency simultaneous full-duplex technology is widely studied as a key $5 \mathrm{G}$ technology. This technology transmits and receives on the same time and frequency resources, and reduces the interference between the transmit and receive links by means of interference cancellation. If the interference can be eliminated ideally, the spectrum efficiency can theoretically be doubled.

(4) Enhanced multi-carrier technology

$5 \mathrm{G}$ has proposed technologies that use different waveforms for different application scenarios, namely through multi-carrier frequency division multiplexing technology, and other alternative multi-carrier technologies, such as filter bank-based multi-carrier FBMC, general filtering Multi-carrier UFMC, etc. These multi-carrier technologies have higher utilization efficiency of physical spectrum and are suitable for spectrum sharing application scenarios, which can greatly improve transmission performance and reduce link access delay.

(5) Flexible duplex technology

In order to adapt to the rapid changes in upstream and downstream services, the flexible duplex mode enhances the flexibility of resource allocation and improves resource utilization. This is particularly important for the current increasingly tight wireless resources. Current research is divided into FDD asymmetric frequency band allocation, TDD flexible resource allocation, and dynamic spectrum allocation.

(6) Advanced coding and modulation technology

In order to make more effective use of limited communication resources to achieve wireless transmission with high throughput, high spectrum efficiency and high quality of service, $5 \mathrm{G}$ adopts new coding technologies: LDPC coding and Polar coding for better error correction. $5 \mathrm{G}$ also uses high-order 1024QAM modulation, which greatly improves the spectrum efficiency. At present, this technology has become a $5 \mathrm{G}$ standard and is suitable for scenarios such as large bandwidth, ultra-low latency and high reliability.

\section{Application of $5 \mathrm{G}$ non-orthogonal technology in power communication system}

In response to the demand for dense mass access and ultralarge capacity of wireless networks, $5 \mathrm{G}$ proposes nonorthogonal multiple access technology to solve this problem.

\subsection{Non-orthogonal multiple access technology}

The industry has conducted research and discussion on a variety of new multiple access technologies, such as Sparse Code Division Multiple Access (SCMA), power division Non-Orthogonal Multiple Access (NOMA), Multi-User Shared Access (MUSA), and Patterns Split Multiple Access (PDMA) technology, etc.

Various non-orthogonal multiple access technologies use nonlinear detection technology to demodulate multiuser signals through repeated iterations. The following is a more detailed introduction of a typical PDMA technology to analyze the feasibility of its application to power multi-terminal connections. Other non-orthogonal multiple access technologies have similar effects.

PDMA is based on the principle of introducing reasonable unequal diversity among multiple users to increase capacity. By designing a multi-user unequal diversity sparse pattern matrix and a codebook codeword optimization scheme for joint coding and modulation, time domain, frequency domain and Multi-dimensional non-orthogonal signal superimposition and transmission in space, etc., is implemented to obtain the higher multiuser multiplexing and diversity gain.

The coding domain of PDMA means that different users perform non-orthogonal user signal superposition by multiplexing different columns of the PDMA pattern matrix on the same time-frequency resource. The corresponding system model can be briefly expressed as:

$$
\begin{gathered}
\mathrm{Y}=\left(\mathrm{H}_{\mathrm{ch}} \otimes \mathrm{H}_{\mathrm{PDMA}}\right) \mathrm{X}+\mathrm{N}=\mathrm{HX}+\mathrm{N} \\
\left(\begin{array}{c}
y_{1} \\
y_{2} \\
\ldots \\
y_{N}
\end{array}\right)=\left(\begin{array}{ccccc}
h_{1,1} & h_{1,2} & 0 & h_{1, m} 2 & 0 \\
h_{2,1} & h_{2,2} & h_{2, m 1} & 0 & 0 \\
\ldots & \ldots & \ldots & \ldots & \ldots \\
h_{N, 1} & 0 & h_{N, m 1} & 0 & h_{N, M}
\end{array}\right) \cdot\left(\begin{array}{c}
x_{1} \\
x_{2} \\
\ldots \\
x_{M}
\end{array}\right)+\left(\begin{array}{c}
n_{1} \\
n_{2} \\
\ldots \\
n_{N}
\end{array}\right)
\end{gathered}
$$

Among them, Y represents the receiving signal vector at receiving end, $\mathrm{N}$ represents the noise vector at receiving end, $\mathrm{X}$ represents the signal vector at sending end, HPDMA represents the PDMA multi-user pattern matrix, $\mathrm{H}_{\mathrm{ch}}$ represents the real wireless channel response matrix, $\mathrm{H}=\left(\mathrm{H}_{\mathrm{ch}} \otimes \mathrm{H}_{\text {PDMA }}\right)$, represents the PDMA multi-user code from the sender to the receiver. The composite equivalent channel response matrix of the matrix and the real wireless channel response matrix, " $\otimes$ " represents the product of the corresponding position elements of the two matrices. $\mathrm{y}_{1}, \mathrm{y}_{2}, \ldots \mathrm{y}_{\mathrm{N}}$ indicates the received signals corresponding to $\mathrm{N}$ time-frequency resource units at receiving end, $\mathrm{n}_{1}, \mathrm{n}_{2}, \ldots \mathrm{n}_{\mathrm{N}}$ indicates the noise corresponding to the $\mathrm{N}$ time-frequency resource units at the receiving end, $\mathrm{x}_{1}, \mathrm{x}_{2}, \ldots \mathrm{x}_{\mathrm{N}}$ indicates the transmission signal corresponding to $\mathrm{M}$ users for resource multiplexing at the transmitting end, $h_{n, m}$ represents the equivalent channel response of the PDMA multi-user coding matrix and the real wireless channel response matrix from the sender to the receiver.

When $\mathrm{M}$ takes the theoretical maximum value of $\mathrm{M}=2^{\mathrm{N}}-1$, the theoretical PDMA multi-user coding matrix $\mathrm{H}_{\text {PDMA }}$ can be expressed in the following form: 


$$
H_{P D M A}^{(N, M)}=\left(\begin{array}{ccccc}
1 & 1 & 0 & 1 & 0 \\
1 & 1 & 1 & 0 & 0 \\
\ldots & \ldots & \ldots & \ldots & \ldots \\
1 & 0 & 1 & 0 & 1
\end{array}\right)_{N \times M}
$$

The actual value of $M$ depends on the desired multiplexing multiple and the system implementation complexity. Therefore, the actual PDMA coding matrix $\mathrm{H}_{\mathrm{PDMA}}^{(\mathrm{N}, \mathrm{M})}$ comes from the selection of some columns of the theoretical PDMA coding matrix. The basic principle is that there are reasonable inequalities between different columns.

Taking 3 basic time-frequency resource units (Resource Units, RE) in an OFDM system to transmit 5 users as an example, the following figure shows the mapping of the PDMA coding domain to the time- frequency resources, and the corresponding PDMA coding matrix is expressed as:

$$
\mathrm{H}_{\mathrm{PDMA}}^{(3,5)}=\left(\begin{array}{lllll}
1 & 1 & 0 & 0 & 1 \\
1 & 1 & 1 & 0 & 0 \\
1 & 0 & 1 & 1 & 0
\end{array}\right)_{3 \times[5}
$$

User 1 sends data on the 3 REs, user 2 sends data on the 1 st and 2nd REs, user 3 sends data on the 2nd and 3rd REs, and users 4 and 5 send data on the 3 rd and 1 st REs respectively. The first RE that is finally superimposed contains the information of users 1,2 , and 5 , the second RE contains the information of users 1,2 , and 3 , and the third RE contains the information of users 1,3 , and 4 .

The following figure shows an example of PDMA uplink transmission and reception.

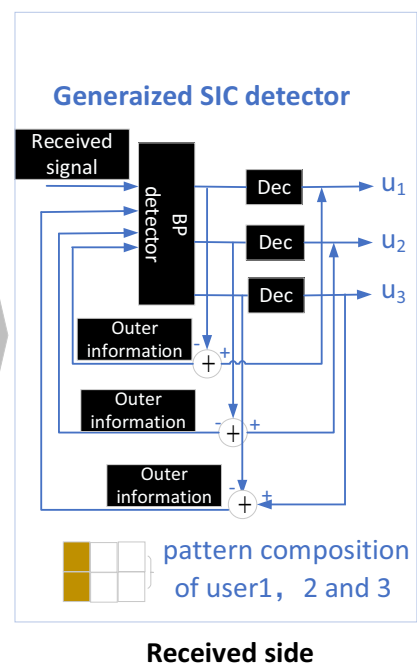

Figure 4. PDMA uplink transmission and receiving

Transmitter end: Multiple user data performs forward error correction channel coding, PDMA coding modulation, PDMA subcarrier resource mapping, and OFDM modulation to complete signal processing at the transmitter.

Receiving end: The received signal undergoes OFDM demodulation and Belief Propagation Iterative Detection and Decoding (BP-IDD), and finally the transmission data of each terminal is obtained. Among them, the BP-IDD algorithm adopted by the receiver is essentially a joint iterative processing of Turbo decoding and BP detection, and a BP detection algorithm without Turbo iteration can also be used.

On the basis of the PDMA coding domain, the superposition of the power domain and the generalized spatial domain can be further considered to optimize the multi-user pattern design. Power domain pattern segmentation is based on user channel quality for power allocation. In theory, each user can occupy all timefrequency resources of the system. The user scheduling algorithm is assisted at the transmitting end, and serial interference cancellation is performed at the receiving end. User capacity, especially the edge user capacity, has been improved.

The generalized spatial pattern division is based on the serial interference cancellation receiver, which performs spatial coding on the user signal, so that the user signal can be effectively divided after being received by the serial interference cancellation receiver, thereby realizing multiple access.

\subsection{PDMA performance analysis}

Compared with Orthogonal Technology (OMA), PDMA technology can not only greatly increase system capacity, accept and schedule more terminals, but also achieve better demodulation performance through reasonable selection of coding matrices and interference iteration processing algorithms.

The PDMA demodulation performance and access capacity are given below through simulation.

(1). Demodulation performance

Simulation conditions: Uma_NLOS channel, $3 \mathrm{~km} / \mathrm{h}$ moving speed, QPSK modulation, $150 \%$ load rate (that is, 3 users occupy 2 resource blocks on average), power ratio $1: 2: 2$, in the three dimensions of time-frequency and power. For user signal superposition, the receiver uses the BP-IDD algorithm. 


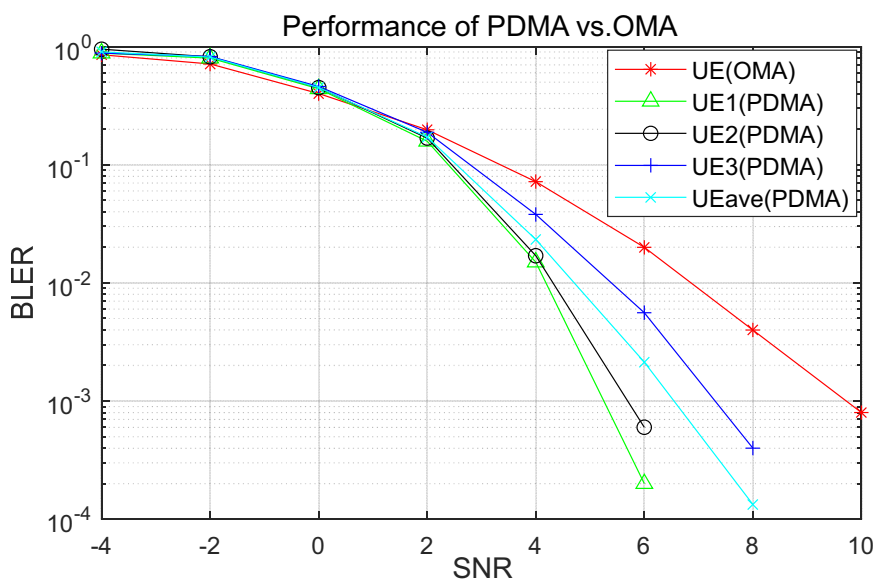

Figure 5. BLER of PDMA vs. OMA

In figure 5, UE1, UE2, and UE3 respectively represent the block error rate of 3 users using the PDMA mode, $\mathrm{UE}_{\mathrm{ave}}$ represents the average block error rate of 3 users, and UE (OMA) represents the single user block error rate using the orthogonal mode. It can be seen from figure 5 that by reasonably selecting the PDMA coding combination and using the efficient BP-IDD algorithm, each multiple access user in the non-orthogonal PDMA multiple access method can obtain better performance than the traditional orthogonal multiple access method. Among them, the difference in the receiving performance of the three users is related to the user's transmit power, the degree of resource reuse, and the demodulation sequence.

(2). System capacity

Simulation conditions: Uma NLOS channel, $3 \mathrm{~km} / \mathrm{h}$ moving speed, QPSK modulation, 200\% load rate (that is, an average of 6 users occupy 3 resource blocks), equal power ratio, user signal superimposition in the three dimensions of time-frequency and power, the receiver uses the BP-IDD algorithm.

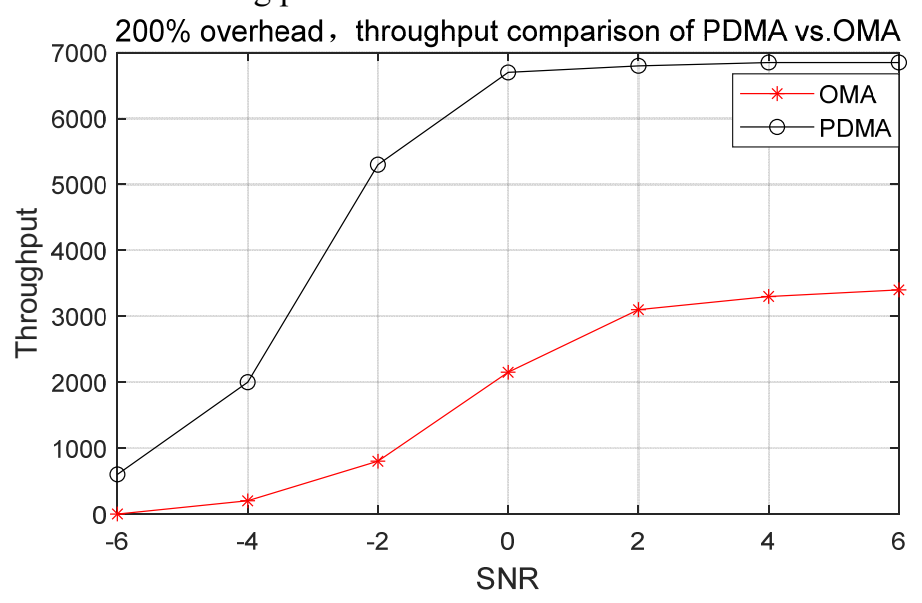

Figure 6. throughput of PDMA vs. OMA

In figure 6, the OMA and PDMA curves represent the system throughput rates when orthogonal and nonorthogonal access modes are used, respectively. It can be seen from the figure that the total throughput of PDMA is approximately twice that of OMA.

From the above simulation of demodulation performance and throughput rate, it can be seen that by controlling the overload rate and using more advanced receiver algorithms, non-orthogonal PDMA technology can not only access more end users, but also achieve better reception performance.

\subsection{Application of PDMA technology on smart grid}

The smart grid covers power generation, transmission, transformation, distribution, and utilization. There are many types of businesses. Distributed power generation, electricity consumption information collection and other businesses urgently need large-connected networks to support. Information collection services mainly include useful electricity information collection, electric vehicle charging stations/piles, power quality monitoring, comprehensive monitoring of power distribution stations, etc. This type of business involves massive communication terminals and is widely distributed in all links of the power grid, with typical wide coverage and big connection business characteristics, the connection density is tens of thousands per square kilometre. In the future, the collection frequency will tend to be on the minute level, and the number of connections will at least double. In the direct copy mode, the number of connections is expected to increase by $50-100$ times, and 
the demand for connections will reach billions.

- For the grid information collection scene, analyze its communication characteristics:

- There are a large number of connection terminals, including both regular power supply equipment and battery-powered sensor equipment;

- In generally, no high-speed mobility, purely static or quasi-static network topology in smart grid;

- The transmission rate is not too high, but the service frequency is high, and the multi-terminal concurrency is strong;

- Wide coverage and strong requirements for receiving sensitivity;

- The upstream transmission service is mainly used, and the downstream is mainly control information.

PDMA typically has the characteristics of large connections and high performance, and has important value in increasing the capacity and coverage of energy information collection networks. However, it is necessary to pay attention to and solve some specific problems in applying this technology to integrated energy information collection and communication systems such as electric power. The principles are as follows:

(1). Complexity

Power communication terminals generally require low power consumption and low cost, especially batterypowered sensing equipment, while PDMA demodulation requires nonlinear iterative processing, which requires certain computing and storage capabilities of the processor. PDMA technology can only be applied to some relay nodes with strong power supply, computing and storage capabilities.

(2). Application mode

On the one hand, PDMA requires centralized scheduling and control. On the other hand, power information collection is mainly for upstream service transmission. Therefore, it can be considered to use PDMA demodulation only in the upstream direction and with certain processing capabilities. The collection terminal only needs to modulate and send the PDMA signal according to the scheduling requirements of its aggregation terminal.

The following figure shows a typical tree topology diagram of the power acquisition system, where $\mathrm{A}$ is the centralized controller, which is responsible for controlling the collection of information of all nodes under it and reporting to the central control unit. $\mathrm{B}$ and $\mathrm{C}$ are the first and second level aggregation nodes, and $\mathrm{D}$ is the end node. Generally speaking, battery-powered sensor devices can only be used as Class D nodes.

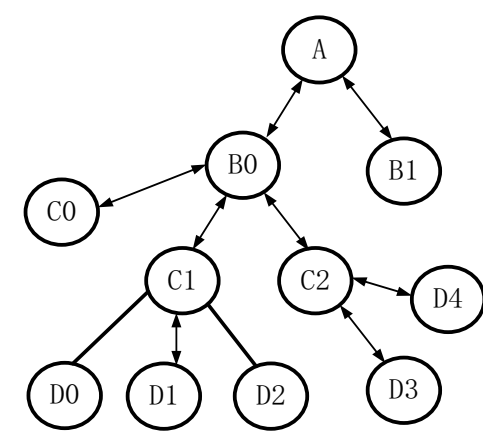

Figure 7. typical tree topology diagram of the power acquisition system

Both the aggregation node and the end node have information collection capabilities, and the aggregation node also has the information aggregation relay capability of its subordinate nodes. From A to other nodes is called downlink transmission, and vice versa is uplink transmission.

Based on the topology shown in figure 7 and the PDMA application principles analyzed above, consider applying PDMA access only in the uplink direction. The centralized control node and the aggregation node perform resource scheduling, and receive and demodulate the PDMA signal, while the end node only needs to send simple PDMA signals. For example, in the figure A, B0, $\mathrm{C} 1$ and $\mathrm{C} 2$ use PDMA scheduling and demodulation processing, and other nodes only need to obey the scheduling and modulation processing.

In summary, it is feasible to effectively apply PDMA technology to grid information collection. Applying PDMA technology to only some nodes with processing capabilities and a large number of subordinate node scheduling can increase the access capacity of the entire network, but will not increase the power consumption and cost of weak nodes. At the same time, considering the fact that the physical topology of the actual scene is static or quasi-static and the real-time requirements are not too high, the use of more iterative demodulation processes can further improve the system's receiving performance and network capacity.

\section{Conclusion}

In the future, power information collection will mainly develop in the direction of increasing collection frequency, rich collection content, and two-way interaction. The frequency of collection is increased, in order to more effectively achieve peak-shaving and valley-filling, and to support more flexible stepped pricing, the metering interval will be increased from the current hourly level to the minute level to achieve quasi-real-time data information feedback. The collection content is rich. For household users, in the future, in addition to the overall electricity consumption information of the electricity consumption household, the collection content will be extended to the indoor network (HAN) in the user's residence to realize the information metering of the indoor electric equipment. In addition, as distributed power 
sources, electric vehicles, energy storage devices and other user-side equipment are connected to the grid in a two-way manner, the scope of grid measurement and observation will be further expanded.

As a large number of collection terminals need to access the network, and concurrency increases due to the increase in collection frequency, there is an urgent need to improve multi-user access capabilities. 5G technology includes mobile internet scenarios for large bandwidth, industrial interconnection scenarios for low latency and high reliability, and machine communication scenarios for massive connections. Various wireless air interface technologies and wireless network technologies developed in the early stages are being widely used in various industries such as 3GPP standards, $5 \mathrm{G}+$ industry, and $5 \mathrm{G}+$ agriculture. Among them, the non-orthogonal multiple access technology is a research to solve the problem of massive user access, which meets the needs of a large number of terminal information collection and reporting requirements of the power communication system.

This article analyzes the principle and performance of 5G non-orthogonal multiple access technology with PDMA technology as an example, and gives the principle and basic scheme of technology application in combination with power communication business requirements and actual communication characteristics. Through the practical application of this technology, it is possible to increase the capacity of the electric power communication network, strengthen the wide and deep coverage of the wireless network, reduce the business delay, fully and deeply perceive the operation, status and environmental information of the source network and load storage equipment, optimize the power dispatch and control, and achieve improvement New energy consumption capacity.

In summary, the technical application described in this article is not only applicable to power acquisition and communication scenarios, but also can be applied to various energy system information transmission networks.

\section{Acknowledgments}

We would like to express our gratitude to Smart Shine Microelectronics LTD. who provided strong financial support for this article, and to the conference organizers who provided technical support for the publication of this article.

\section{References}

1. Erik Dahlman, Stefan Parkvall, Johan Skold, 5G NR Standard: The Next Generation Wireless Access Technology, China Machine Press, 2019.5

2. Wang Yingmin, Sun Shaohui, Gao Qiubin: 5G Transmission Key Technology, Publishing House of Electronics Industry, 2017.3. (in Chinese)

3. State Grid Shandong, 5G Application Scenarios Analysis on Grid Industry, 2020.7.
4. Chongqing University of Posts and Telecommunications, Research on 5G NonOrthogonal Access System PF Scheduling Strategy 2017.1.

5. G.Boudreau, J.Panicker, N.Guo, R.Chang, N.Wang, and S.Vrzic. "Interference coordination and cancellation for $4 \mathrm{G}$ network" IEEE Communications Magazine, 2009,47(4):74-81.

6. Y. Wu, S. Zhang, Y. Chen. "Iterative multiuser receiver in sparse code multiple access systems". IEEE International Conference on Communications, 2015, pp.4521-4526.

7. X. Dai, S. Sun, and Y. Wang, "Successive interference cancellation amenable space-time codes with good multiplexing-diversity tradeoffs", 15 th Asia-Pacific Conference on Communications, APCC 2009.

8. Z. Yuan, G. Yu, and W. Li, "Multi-User Shared Access for 5G Telecommun. Network Technology", vol. 5, no. 5, May 2015, pp. 28-30.

9. D. Truhachev, "Achieving AWGN multiple access channel capacity with spatial graph coupling", IEEE Communications Letters, 2012, 16(5):585588.

10. China Academy of Telecommunications Technology, "PDMA: Pattern Division Multiple Access Technology for 5G".

11. 3GPP, TS 38.211 V15.5.0 Technical Specification, 2019.

12. 3GPP, TS 38.212 V15.5.0 Technical Specification, 2019

13. H. Nikopour, E.Yi, A. Bayesteh, et al. "SCMA for downlink multiple access of $5 \mathrm{G}$ wireless networks". IEEE Global Communications Conference, 2014, pp. 3940-3945. 